\title{
LA REVUE CANADIENNE DE SANTÉ MENTALE COMMUNAUTAIRE DE 1982 À 2006: UNE ANALYSE DE CONTENU
}

\author{
LAURENCE FORTIN-PELLERIN, JOËLLE POULIOT-LAPOINTE, \\ CAROLINE THIBODEAU et MARIE-HÉLÈNE GAGNÉ \\ École de psychologie, Université Laval, Québec
}

\begin{abstract}
RÉSUMÉ
Cet article présente une analyse de contenu des articles publiés dans la Revue canadienne de santé mentale communautaire (RCSMC) de 1982 à 2006. Cette analyse vise à dresser un portrait de l'ensemble des articles, à dégager les grandes tendances notables au fil du temps et à procurer des pistes de réflexion pour les futures publications de la RCSMC. Différentes dimensions sont présentées et discutées dans une perspective d'évolution temporelle, soit l'identité et la provenance des rédacteurs et rédactrices et des auteurs les plus productifs, la langue de publication, l'affiliation des premiers auteurs des articles, les thématiques des numéros spéciaux et des suppléments, le type et le domaine d'intérêt des articles et les mentions de financement pour des recherches empiriques publiées dans la RCSMC. Ces résultats sont discutés à la lumière des connaissances historiques et actuelles en santé mentale communautaire.
\end{abstract}

Le rôle des revues scientifiques consiste à fournir un moyen de communication entre chercheurs*, de même qu'entre chercheurs et professionnels et professionnelles. Elles sont notamment utilisées pour présenter les résultats de recherches qui relèvent d'un champ d'expertise particulier. Les articles publiés dans ces revues permettent de mesurer le progrès d'un domaine scientifique tout en reflétant les intérêts et les préoccupations des professionnels et professionnelles du domaine couvert par la revue, d'où l'intérêt d'analyser leur contenu (Buboltz \& Savickas, 1994).

La Revue canadienne de santé mentale communautaire (RCSMC) énonce sa mission de la façon suivante:

Offrir un lieu d'échange aux Canadiens appartenant aux milieux tant académiques que professionnels et intéressés à l'approche communautaire en santé mentale, particulièrement la promotion de ses

\footnotetext{
*Les termes auteur et chercheur réfèrent sans distinction aux hommes et aux femmes.

Les auteures tiennent à remercier Francine Lavoie et Tim Aubry pour leurs précieux commentaires en cours de rédaction, de même que la Revue canadienne de santé mentale communautaire pour son soutien financier et Jean-Michel Roy pour son soutien technique. Toute correspondance concernant cet article devrait être envoyée à Marie-Hélène Gagné, professeure, École de psychologie, Université Laval, Québec (Québec), G1K 7P4. Courriel: marie-helene.gagne@psy.ulaval.ca
} 
dynamismes positifs et les dimensions curatives et préventives. La RCSMC est un forum interdisciplinaire consacré au partage de l'information et à l'élaboration d'un savoir fiable et valide à propos des phénomènes se rapportant au bien-être psychologique des individus et des communautés au Canada.

Dès ses débuts en 1982, la RCSMC a privilégié la publication d'articles en lien avec les domaines d'intérêt suivants: organisation des services sociaux, évaluation de programme, entraide, éducation communautaire, formation, consultation, action sociale, développement communautaire, analyse des besoins de la communauté et défense de droits et d'intérêts. Le bilinguisme et l'interdisciplinarité de la RCSMC la rendent unique dans le domaine de la santé mentale communautaire au Canada (Revue canadienne de santé mentale communautaire, 2001).

La santé mentale communautaire renvoie à un ensemble d'attitudes et d'idéologies relatives à l'étude des problèmes humains et des solutions à y apporter (Kiely \& Moreau, 1975). Tout en étant spécifiquement concerné par les problèmes de santé mentale (Kiely \& Moreau, 1975), ce domaine étudie à la fois les dynamiques sociales, économiques et culturelles (Bennett \& Trute, 1982) qui favorisent l'émergence et le maintien des problèmes de santé mentale, ou qui, au contraire, les amenuisent. Dans une perspective de santé mentale communautaire, on considère que les carences individuelles sont des manifestations de dysfonctionnement des groupes primaires tels l'école, le milieu de travail et la famille. Ainsi, on se donne pour objectif de réaménager ces réseaux pour pallier aux déficits affichés par les individus (Morency, Couture, Johnson, \& Kiely, 1988). Dans cette optique, la santé mentale communautaire se base sur huit valeurs fondamentales: l'autonomie, la confidentialité, le soutien, la sécurité, la confiance, la participation, la solidarité et la justice (Liégeois \& Audenhove, 2005).

La RCSMC a fait l'objet d'une analyse de contenu en 1993 après 10 ans de publication. Les faits suivants ressortent de cette analyse: (a) les femmes sont minoritaires parmi l'ensemble des auteurs; (b) la majorité des auteurs sont affiliés à une institution universitaire; (c) les articles empiriques sont moins nombreux que les articles non empiriques; (d) parmi les articles empiriques, ceux qui rapportent une étude où les chercheurs sont face-à-face avec les participants et participantes lors de la collecte des données sont les plus fréquents; et (e) une analyse de niveau individuel se retrouve le plus souvent dans les articles mais plus de la moitié des articles incluent plusieurs niveaux systémiques d'analyse (Peirson \& Walsh-Bowers, 1993).

Pour faire suite à la première analyse et pour souligner le $25^{\mathrm{e}}$ anniversaire de la RCSMC, le but de la présente étude est de dresser un portrait de l'ensemble des articles publiés entre 1982 et 2006, de dégager les tendances notables au fil du temps et de procurer des pistes de réflexion pour les futures publications. C'est lors de sa réunion annuelle tenue en mai 2006 que l'équipe éditoriale de la RCSMC a élaboré ce projet et décidé de soutenir sa réalisation. Le travail d'analyse et de rédaction a été confié à trois étudiantes au doctorat en psychologie communautaire de l'Université Laval.

\section{MÉTHODE}

Afin de dresser un portrait exhaustif des publications de la RCSMC, une analyse de contenu a été effectuée. L'analyse de contenu se définit comme un ensemble de méthodes d'analyse de documents 
textuels permettant d'en expliciter les sens ou les manières dont les contenus parviennent à faire sens. Il existe deux principaux types d'analyse de contenu: quantitative, où l'analyste procède à des codages, des comptages, des comparaisons et où des tendances sont établies; et qualitative, où l'analyste tente d'expliquer la signification exacte d'un message (Mucchielli, 1996). L'analyse de contenu quantitative a été retenue puisqu'elle s'avère particulièrement utile lorsqu'il y a une grande quantité de matériel à analyser (L'Écuyer, 1990), ce qui est le cas pour le contenu des 25 ans de publication de la RCSMC. Ce type d'analyse donne un ordre de grandeur quant à la répartition du matériel dans les différentes catégories d'analyse, en plus de permettre la comparaison quantitative entre divers éléments ou périodes de temps et d'aider à éviter l'égarement (L'Écuyer, 1990).

\section{Corpus de données}

Les données qui sont à la base de la présente analyse sont tirées de l'ensemble des résumés des 495 articles publiés dans la RCSMC de 1982 au printemps 2006, incluant ceux provenant des numéros spéciaux $(n=236)$ et des suppléments $(n=49)$. À l'occasion, le corps des articles a été consulté afin de confirmer ou compléter certaines informations. L'affiliation institutionnelle du premier auteur et la présence ou la mention de financement ne figurant pas dans le résumé, ces informations ont été colligées ailleurs, généralement sur la première page de l'article. Les articles servant d'introduction aux numéros spéciaux, les comptes rendus de livres et les symposiums présentant le point de vue d'auteurs invités à se prononcer sur un sujet commun ont été exclus du corpus de données.

\section{Élaboration de la grille de codification et procédures d'analyse}

Une grille de codification du matériel a été développée en tenant compte à la fois du projet initial validé par l'équipe de rédaction de la RCSMC et des informations disponibles dans les articles. Une version préliminaire de la grille a d'abord été prétestée par les trois analystes. À l'aide de cet outil, elles ont extrait les données de huit résumés de façon indépendante tout en se consultant en cas d'hésitations. Ce prétest a permis de constater que les disciplines des auteurs ayant publié dans la RCSMC ne pourraient pas faire l'objet d'une analyse systématique puisque cette information n'est pas colligée par la RCSMC. Il en va de même pour le genre des auteurs qui est trop souvent difficile à déterminer sur la base du seul nom, d'autant plus que dans certains cas seules les initiales étaient rapportées. C'est pourquoi la discipline et le genre des personnes impliquées dans la RCSMC ont été analysés uniquement pour les rédacteurs et rédactrices et pour les auteurs les plus prolifiques.

En cours d'analyse, la grille a subi d'autres modifications. La dimension «population cible » a dû

être abandonnée. Étant donné la grande diversité des populations ciblées dans les 495 articles, les données étaient difficilement analysables que ce soit de façon quantitative ou qualitative. Il en va de même pour la source de financement des recherches rapportées dans les articles de type empirique. Seule la mention de financement a été considérée au lieu de la source de financement. La grille finale est fournie en appendice.

À l'aide de cette grille, les données ont été codées par les trois analystes relativement aux dimensions suivantes: identité des auteurs, affiliation institutionnelle du premier auteur, type d'article, domaine 
d'intérêt énoncé dans la mission de la RCSMC couvert par l'article, langue dans laquelle l'article est rédigé et mention de financement indiquée des articles de type empirique. Les thèmes des numéros spéciaux et des suppléments, de même que l'identité des rédacteurs et rédactrices, ont aussi été recensés. Plusieurs de ces informations sont de nature factuelle et n'ont pas nécessité le jugement des analystes. Par contre, d'autres dimensions de l'analyse ont nécessité des outils ou des procédures plus élaborés.

Premièrement, la productivité des auteurs ayant publié dans la RCSMC a été déterminée à partir de la formule mathématique de Howard, Cole et Maxwell (1987). Celle-ci a été utilisée dans plusieurs analyses de contenu des publications de revues (Buboltz, Miller, \& Williams, 1999; Cokley, Caldwell, Miller, \& Muhammad, 2001; Loveland, Buboltz, Schwartz, \& Gibson, 2006; Pope-Davis, Ligiero, Liang, \& Codrington, 2001) et permet de tenir compte à la fois du nombre d'auteurs et de leur rang dans la publication. Pour un même article, les pointages associés à chaque auteur totalisent toujours 1 point et s'ajustent selon le nombre d'auteurs. À titre d'exemple, si un article est rédigé par un seul auteur, celui ou celle-ci se voit attribuer le pointage 1 . S'il est écrit par deux auteurs, le premier ou la première reçoit le pointage 0,6 et le ou la deuxième 0,4. La formule s'applique à un nombre infini d'auteurs.

Deuxièmement, chacun des articles a été catégorisé par type de publication: (a) empirique (qualitatif ou quantitatif), (b) communautaire pratique, (c) recension d'écrits ou (d) théorique. Ces dimensions ont été établies à partir des principaux types d'articles habituellement publiés dans les analyses de contenu de revues communautaires (Novaco \& Monahan, 1980; Peirson \& Walsh-Bowers, 1993). En cas de doute à savoir dans quelle catégorie classer un article donné, les analystes procédaient par consensus. Les définitions opérationnelles de chacune des catégories sont fournies en appendice.

Enfin, les domaines d'intérêt énoncés dans la mission de la RCSMC ont été repris dans l'analyse pour examiner les thèmes couverts par les articles: (a) organisation des services sociaux, (b) évaluation de programme, (c) analyse des besoins de la communauté, (d) développement communautaire, (e) consultation, (f) action sociale, (g) entraide, (h) éducation communautaire et formation et (i) défense de droits et d'intérêts (Revue canadienne de santé mentale communautaire, 2001). Un thème supplémentaire s'est ajouté en cours d'analyse, soit celui de la réaction à un phénomène donné. Les domaines de la formation et de l'éducation communautaire ont été fusionnés, puisque très semblables. Comme pour le type de document, une approche par consensus entre analystes a été appliquée en cas de doute quant à la catégorie la plus appropriée. Les définitions opérationnelles de chacune des catégories sont fournies en appendice.

\section{Comparaison entre la présente analyse de contenu et celle effectuée en 1993}

Certains éléments pris en considération dans la première analyse de contenu effectuée en 1993 (Peirson \& Walsh-Bowers, 1993) l'ont aussi été dans la présente analyse. Il s'agit de l'affiliation des auteurs et du type d'article. Étant donné le nombre important d'articles à traiter $(N=495)$ et la difficulté de traiter ces dimensions uniquement à partir des résumés, les autres dimensions couvertes par la précédente analyse n'ont pas été répliquées dans celle-ci. Il s'agit du genre des auteurs, de la collaboration entre auteurs à des publications communes, du niveau systémique d'analyse, de la population à l'étude et des relations de collaboration entre chercheurs et participants et participantes. Par contre, d'autres dimensions ont été ajoutées à la présente analyse, soit une recension chronologique 
des rédacteurs et rédactrices et des numéros spéciaux et des suppléments, un indicateur de productivité des auteurs ayant publié dans la RCSMC, la présence des domaines d'intérêt énoncés dans la mission de la RCSMC, la langue de publication de l'article et la mention de financement pour les recherches empiriques publiées. Ces dimensions fournissent des informations très pertinentes à savoir « qui » a le plus d'influence sur le développement de la RCSMC et sur son contenu et, justement, quelles sont les principales caractéristiques de ce contenu.

\section{RÉSULTATS}

Identité, productivité et provenance des personnes influentes

Rédactrices et rédacteurs. Les rédactrices et rédacteurs ayant œuvré à la RCSMC depuis ses débuts sont présentés dans le tableau 1. Ces personnes sont responsables entre autres de coordonner la révision des manuscrits en anglais et en français, de guider les rédacteurs et rédactrices des numéros spéciaux de la RCSMC et de participer à plusieurs autres tâches de gestion de la RCSMC. Elles exercent donc une influence majeure sur le contenu de la RCSMC. À ce titre, il est intéressant de noter que la RCSMC a eu 10 rédactrices et 9 rédacteurs au fil du temps, ce qui suggère une équité sur le plan du genre. Ceux-ci proviennent d'une diversité d'établissements universitaires situés dans quatre provinces

\section{Tableau 1}

Rédacteurs et rédactrices de la RCSMC de 1982 à 2006

\begin{tabular}{llll}
\hline Années & Rédacteurs et rédactrices & Discipline & Affiliation \\
\hline $1982-1990$ & Edward M. Bennett & Psychologie & Wilfrid Laurier University \\
$1982-1990$ & Barry Trute & Service social & University of Manitoba \\
1988 & Maurice Payette & Psychologie & Université de Sherbrooke \\
$1989-1992$ & Céline Mercier & Psychologie & Hôpital Douglas \\
$1990-1994$ & Kathryn McCannell & Service social & University of British Columbia \\
$1990-1996$ & Geoffrey Nelson & Psychologie & Wilfrid Laurier University \\
$1992-1995$ & Jacques Rhéaume & Sociologiea & Université du Québec à Montréal \\
$1994-1999$ & Judy Lynam & Sc. infirmières & University of British Columbia \\
$1995-1999$ & Francine Lavoie & Psychologie & Université Laval \\
$1996-2001$ & Ed Pomeroy & Psychologie & Brock University \\
1999 & Jérôme Guay & Psychologie & Université Laval \\
$2000-2001$ & Annie Devault & Psychologie & Université du Québec à Hull \\
$2000-2002$ & Paule McNicoll & Service social & University of British Columbia \\
$2002-2004$ & Bernadette Dallaire & Service social & Université Laval \\
$2002-2005$ & Diane Hiebert-Murphy & Service social & University of Manitoba \\
$2003-2005$ & Marshall Fine & Service social & Wilfrid Laurier University \\
$2005-2006$ & Marie-Hélène Gagné & Psychologie & Université Laval \\
2006 & Tim Aubry & Psychologie & University of Ottawa \\
2006 & Terry Krupa & Réadaptation & Queen’s University \\
\hline
\end{tabular}

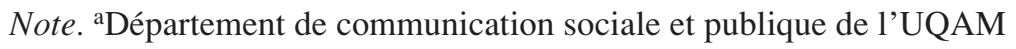


différentes, la plupart en Ontario et au Québec. Sur le plan des disciplines, la psychologie $(n=10)$ et le service social $(n=6)$ semblent surreprésentées.

Auteurs. Depuis 1982, un total de 821 auteurs différents ont publié dans la RCSMC. Le tableau 2 présente les 11 auteurs les plus prolifiques au cours de cette période (deux d'entre eux arrivent ex aequo au $10^{\mathrm{e}}$ rang). Parmi ceux-ci, on constate une représentation assez équitable des hommes et des femmes, bien qu'il n'ait pas été possible de vérifier si cette équité s'observe pour l'ensemble des auteurs. Les plus prolifiques proviennent de divers établissements universitaires situés dans quatre provinces différentes, surtout en Ontario et au Québec. Ce résultat se reflète aussi dans le tableau 3, qui présente les fréquences et pourcentages des cinq principales affiliations institutionnelles de l'ensemble des premiers auteurs des articles publiés. Il reste que près des trois quarts des articles ont pour premier auteur quelqu'un qui provient d'une autre université ou organisation canadienne ou américaine, ce qui suggère une grande diversité de provenance des auteurs. Finalement, les affiliations à des institutions universitaires totalisent 70,1\% de l'ensemble des affiliations de premiers auteurs. Les affiliations institutionnelles non universitaires représentent moins du tiers de la production.

Tableau 2

Les auteurs les plus productifs

\begin{tabular}{llllrr}
\hline Rang & Discipline & Auteur & Affiliation & $\begin{array}{c}\text { Nombre } \\
\text { d'articles }\end{array}$ & Poids \\
\hline 1 & Psychologie & Geoffrey Nelson & Wilfrid Laurier University & 13 & 5,7 \\
2 & Psychologie & Stewart Page & University of Windsor & 5 & 3,6 \\
3 & Psychologie & Camil Bouchard & Université du Québec à Montréal & 5 & 3,4 \\
4 & Psychologie & Céline Mercier & Hôpital Douglas & 5 & 3,3 \\
5 & Sociologie & Françoise Boudreau & York University & 4 & 3,2 \\
6 & Psychologie & Sylvie Jutras & Université du Québec à Montréal & 4 & 2,9 \\
7 & Service social & Christopher Bagley & University of Calgary & 4 & 2,7 \\
8 & Service social & Leslie M. Tutty & University of Calgary & 4 & 2,6 \\
9 & Psychologie & Francine Lavoie & Université Laval & 6 & 2,6 \\
10 & Service social & Barry Trute & University of Manitoba & 5 & 2,5 \\
10 & Psychologie & Edward M. Bennett & Wilfrid Laurier University & 4 & 2,5 \\
\hline
\end{tabular}

\section{Tableau 3}

Principales affiliations institutionnelles des premiers auteurs

\begin{tabular}{lcc}
\hline Affiliation & Proportion (\%) & Fréquence \\
\hline Wilfrid Laurier University & 6,5 & 32 \\
Université de Montréal & 6,1 & 30 \\
Université Laval & 5,5 & 27 \\
University of Toronto & 4,6 & 23 \\
Université du Québec à Montréal & 4,4 & 22 \\
\hline
\end{tabular}




\section{Langue de publication}

La langue dans laquelle l'article est rédigé a été compilée pour 490 articles. Les cinq autres articles sont tirés d'un numéro spécial complètement bilingue: tous les articles y ont été publiés dans les deux langues. Les trois quarts des articles unilingues sont rédigés en anglais et le quart en français. Durant les 5 premières années de publication, les articles francophones représentaient 13\% du nombre total d'articles. Cette proportion a augmenté rapidement et se maintient à environ 30\% jusqu'à maintenant, avec 29,5\% entre 1987 et 1996, 33,8\% entre 1997 et 2001 et 27,5\% entre 2002 et 2006. La figure 1 illustre ce résultat.

Figure 1

Évolution de la langue des articles par tranche de 5 ans

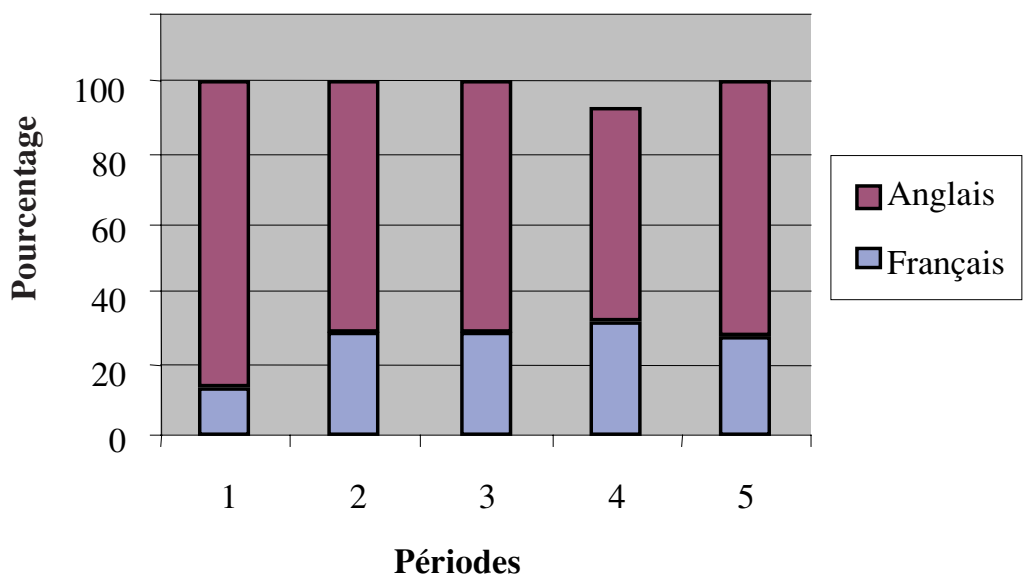

Note. $1=1982-86 ; 2=1987-91 ; 3=1992-96 ; 4=1997-2001 ; 5=2002-6$.

\section{Thèmes abordés et types d'articles publiés}

Numéros spéciaux et suppléments. À chaque année, un numéro spécial a été publié dans la RCSMC. Le thème de ce numéro est choisi par l'équipe de rédaction, qui fait ensuite appel à des rédactrices et rédacteurs invités pour prendre en charge le processus de publication. Aussi, quatre suppléments ont été publiés: des actes de colloque ou d'atelier pertinents au domaine de la santé mentale communautaire (1983 et 1998), un rapport de recherche sur le thème de la législation en santé mentale et des attitudes de la population (1991) et les résultats d'une équipe de recherche sur les transitions familiales (2002).

Les titres des numéros spéciaux et des suppléments des 25 années de la RCSMC sont présentés dans le tableau 4. Il s'avère que près de la moitié des numéros traitent directement de la santé mentale. Plusieurs domaines d'intérêt de la RCSMC sont représentés dans ces numéros, comme par exemple la diversité culturelle, la violence faite aux femmes, les jeunes contrevenants et contrevenantes ou les minorités sexuelles. 


\section{Tableau 4}

\section{Titres des numéros spéciaux et des suppléments de la RCSMC de 1982 à 2005}

\begin{tabular}{|c|c|}
\hline Années & Titres \\
\hline 1982 & Community psychology in Canada \\
\hline \multirow[t]{2}{*}{1983} & Family health practice in Canada \\
\hline & $\begin{array}{l}\text { Supplément spécial: Les répercussions psychosociales de la mise en valeur des richesses naturelles au } \\
\text { Canada: stratégies de recherche et applications }\end{array}$ \\
\hline 1984 & Éducation et formation dans les services communautaires canadiens \\
\hline 1985 & L'évaluation de programme: une étude participative \\
\hline 1986 & Les femmes et la santé mentale \\
\hline 1987 & $\begin{array}{l}\text { Les services de santé mentale communautaire pour les personnes atteintes de troubles mentaux } \\
\text { chroniques }\end{array}$ \\
\hline 1988 & Le phénomène de femmes battues au Canada \\
\hline 1989 & Politique publique, développement économique et social et pouvoir des idées \\
\hline 1990 & Être handicapé au Canada dans les années ' 90 \\
\hline \multirow[t]{2}{*}{1991} & Le support social pour les personnes âgées et leurs aidants \\
\hline & Supplément spécial: Attitudes communautaires et la loi en santé mentale \\
\hline 1992 & Travail, vie privée et santé mentale: le rôle nouveau du communautaire \\
\hline 1993 & La diversité culturelle: la parole, l'accès et la participation \\
\hline 1994 & Prévention: priorité aux enfants et à la jeunesse \\
\hline 1995 & Groupes d'entraide et groupes de soutien \\
\hline 1996 & Le pouvoir, l'oppression et la santé mentale \\
\hline 1997 & La santé mentale dans les communautés autochtones \\
\hline \multirow[t]{2}{*}{1998} & Perspectives critiques de la déontologie appliquée \\
\hline & Supplément spécial: L'évaluation de la qualité de vie en santé mentale \\
\hline 1999 & Innovations positives en santé mentale \\
\hline 2000 & La santé mentale communautaire au Canada, notre passé, notre avenir \\
\hline 2001 & Les jeunes contrevenants et contrevenantes: trouver un juste équilibre entre contrôle et traitement \\
\hline \multirow[t]{2}{*}{2002} & L'innovation en santé mentale communautaire: perspectives internationales \\
\hline & Supplément spécial: Les transitions familiales associées à la séparation des parents \\
\hline 2003 & La norme bouleversée: la santé mentale chez les minorités sexuelles \\
\hline 2004 & La mondialisation et la santé mentale communautaire \\
\hline 2005 & Vieillissement et santé mentale \\
\hline
\end{tabular}


Types d'articles. Plus de la moitié $(54,3 \%)$ des articles publiés dans la RCSMC sont de type empirique. Les articles théoriques constituent le deuxième type d'article le plus fréquent (30,7\%), suivi des articles communautaires pratiques $(8,5 \%)$ et des recensions d'écrits $(6,5 \%)$. La proportion d'articles empiriques a augmenté au fil des années, passant de $34 \%$ au cours des 5 premières années (1982-1986) à 75\% pendant les 5 dernières années (2002-2006). La figure 2 illustre cette tendance.

Figure 2

Évolution du type d'article par tranche de $\mathbf{5}$ ans

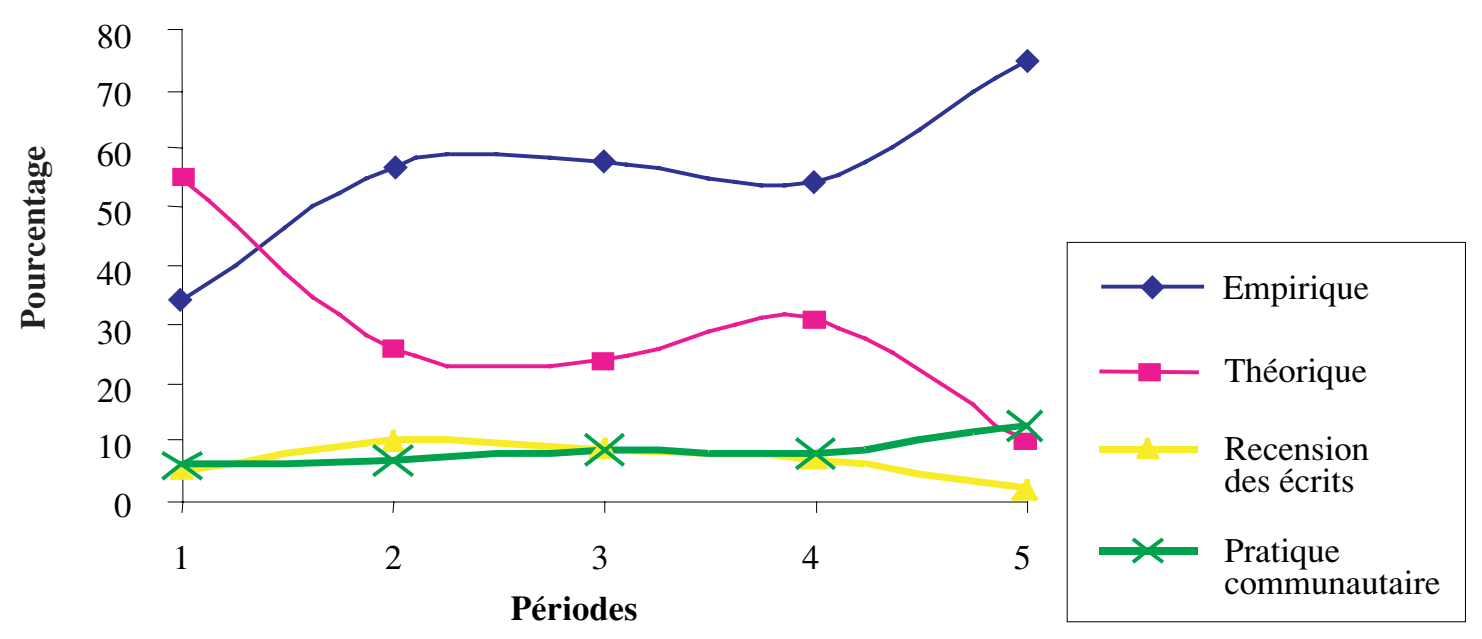

Note. $1=1982-86 ; 2=1987-91 ; 3=1992-96 ; 4=1997-2001 ; 5=2002-6$.

Le tableau 5 présente les types d'articles en fonction de l'affiliation institutionnelle du premier auteur (universitaire vs. non universitaire). Il montre que $79 \%$ des articles empiriques et $81 \%$ des recensions d'écrits scientifiques proviennent d'universitaires. Toutefois, la proportion d'auteurs universitaires diminue considérablement dans le cas des articles communautaires pratiques (52\%) et des articles théoriques (57\%).

Parmi les 269 articles empiriques publiés, les types de méthodologies employées ont été compilés. Avec les années, il y a eu une augmentation régulière de publication de recherches qualitatives, parallèlement à une diminution régulière de publication de recherches quantitatives, comme l'illustre la figure 3. Au cours des 5 dernières années, les deux types de méthodologie sont sur un pied d'égalité au sein de la RCSMC.

Domaines d'intérêt des articles. L'évaluation de programme $(n=79)$ et l'action sociale $(n=78)$ représentent les domaines d'intérêt ayant inspiré le plus grand nombre d'articles. La défense de droits et d'intérêts $(n=24)$, l'entraide $(n=16)$ et la consultation $(n=0)$ sont les domaines d'intérêt les moins couverts par les articles. La popularité des divers domaines d'intérêt varie sensiblement selon la période 
REVUE CANADIENNE DE SANTÉ MENTALE COMMUNAUTAIRE

\section{Tableau 5}

Proportion des premiers auteurs ayant une affiliation universitaire par type d'article

\begin{tabular}{|c|c|c|c|c|}
\hline & $\begin{array}{l}\text { Affiliation } \\
\text { universitaire } \\
\quad n=347\end{array}$ & $\begin{array}{l}\text { Affiliation non } \\
\text { universitaire } \\
\quad n=148\end{array}$ & $\chi^{2}$ & $\mathrm{dl}$ \\
\hline Empirique $(n=269)$ & $79 \%$ & $21 \%$ & \multirow[b]{2}{*}{$23,18 * *$} & \multirow[b]{2}{*}{1} \\
\hline Autre qu'empirique & $59 \%$ & $41 \%$ & & \\
\hline Communautaire pratique $(n=42)$ & $52 \%$ & $48 \%$ & \multirow{2}{*}{$6,88 * *$} & \multirow[b]{2}{*}{1} \\
\hline Autre que communautaire pratique & $72 \%$ & $28 \%$ & & \\
\hline Théorique $(n=152)$ & $57 \%$ & $43 \%$ & \multirow[b]{2}{*}{$19,14 * *$} & \multirow[b]{2}{*}{1} \\
\hline Autre que théorique & $76 \%$ & $24 \%$ & & \\
\hline Recension d'écrits ( $n=32$ ) & $81 \%$ & $19 \%$ & \multirow[t]{2}{*}{2,03} & \multirow[t]{2}{*}{1} \\
\hline Autre que recension d'écrits & $69 \%$ & $31 \%$ & & \\
\hline
\end{tabular}

Note. ${ }^{* *} p<0,01$.

Figure 3

Évolution des méthodologies utilisées dans les articles empiriques par tranche de $\mathbf{5}$ ans

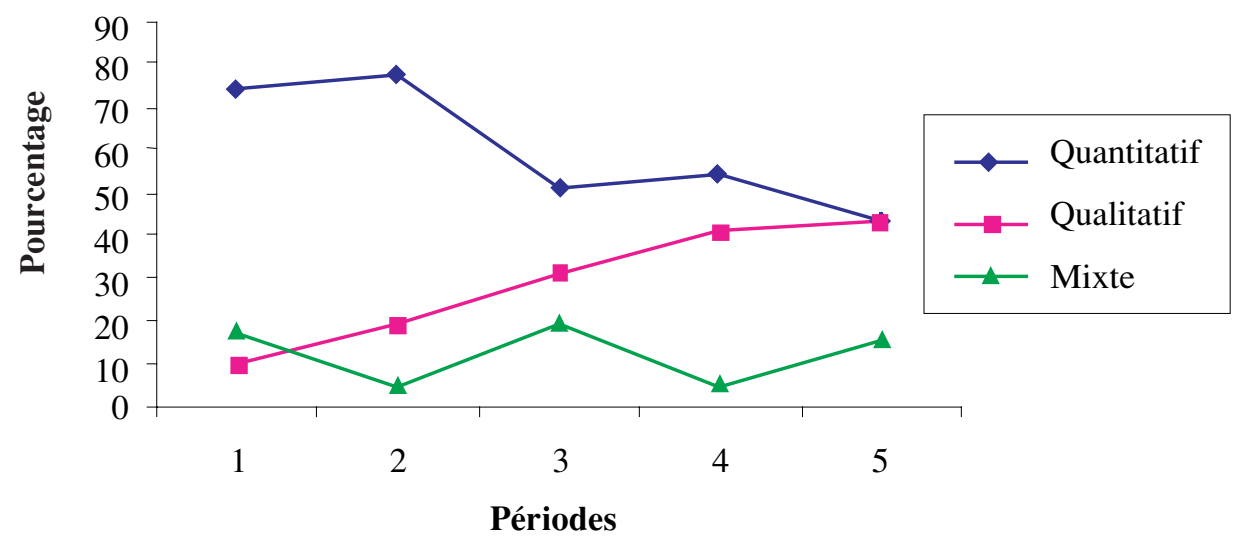

Note. $1=1982-86 ; 2=1987-91 ; 3=1992-96 ; 4=1997-2001 ; 5=2002-6$. 
de temps, comme le montre le tableau 6. D'abord, l'analyse des besoins semble particulièrement populaire entre 1987 et 1991, alors que les évaluations de programmes connaissent une recrudescence entre 1992 et 1996. Au même moment, les articles traitant d'action sociale sont moins représentés que pendant les autres périodes. L'entraide croît en popularité entre 1987 et 1996, puis disparaît complètement. L'éducation communautaire et la formation sont davantage traitées dans les 5 premières années que durant les quatre périodes subséquentes. Enfin, les réactions à un phénomène donné connaissent une baisse entre 1997 et 2001 pour revenir en force entre 2001 et 2006, alors que le thème de la défense de droits et d'intérêts tombe presque à zéro dans les derniers 5 ans.

\section{Tableau 6}

Domaines d'intérêt couverts par les articles de la RCSMC par tranche de 5 ans

\begin{tabular}{|c|c|c|c|c|c|}
\hline & $\begin{array}{c}1 \\
n=123\end{array}$ & $\begin{array}{c}2 \\
n=95\end{array}$ & $\begin{array}{c}3 \\
n=101\end{array}$ & $\begin{array}{c}4 \\
n=85\end{array}$ & $\begin{array}{c}5 \\
n=91\end{array}$ \\
\hline Organisation des services sociaux $(n=47)$ & $6 \%$ & $11 \%$ & $6 \%$ & $14 \%$ & $13 \%$ \\
\hline Évaluation de programme $(n=79)$ & $15 \%$ & $10 \%$ & $27 \%$ & $13 \%$ & $15 \%$ \\
\hline Analyse des besoins de la communauté $(n=32)$ & $4 \%$ & $15 \%$ & $5 \%$ & $6 \%$ & $3 \%$ \\
\hline Développement communautaire $(n=25)$ & $6 \%$ & $7 \%$ & $3 \%$ & $7 \%$ & $2 \%$ \\
\hline Consultation $(n=0)$ & 0 & 0 & 0 & 0 & 0 \\
\hline Action sociale $(n=78)$ & $15 \%$ & $17 \%$ & $7 \%$ & $24 \%$ & $18 \%$ \\
\hline Entraide $(n=16)$ & 0 & $4 \%$ & $12 \%$ & 0 & 0 \\
\hline Éducation communautaire et formation $(n=25)$ & $15 \%$ & $2 \%$ & $2 \%$ & $2 \%$ & 0 \\
\hline Défense de droits et d'intérêts $(n=24)$ & $4 \%$ & $6 \%$ & $7 \%$ & $5 \%$ & $1 \%$ \\
\hline Réaction à un phénomène donné $(n=45)$ & $7 \%$ & $9 \%$ & $10 \%$ & $1 \%$ & $19 \%$ \\
\hline Autres $(n=124)$ & $29 \%$ & $18 \%$ & $22 \%$ & $28 \%$ & $29 \%$ \\
\hline
\end{tabular}

Note. $1=1982-86 ; 2=1987-91 ; 3=1992-96 ; 4=1997-2001 ; 5=2002-6$.

Il reste que le quart des articles publiés ne couvrent pas spécifiquement l'un ou l'autre des domaines d'intérêts identifiés par la mission de la RCSMC. Ils ne correspondent pas non plus à une « réaction à un phénomène donné », une catégorie ayant émergé en cours d'analyse. Ces articles abordent généralement des problématiques plus spécifiques telles la réinsertion sociale, la toxicomanie, la violence, le suicide, etc. Par ailleurs, la majorité de ces articles présentent une composante liée à la santé mentale.

\section{Mention de financement pour les articles empiriques}

La majorité des articles empiriques publiés dans la RCSMC (57\%) ne mentionnent aucune source de financement de la recherche. La proportion d'articles empiriques pour lesquels une mention de financement est présente varie selon la méthodologie utilisée, que ce soit une recherche quantitative (46\%), qualitative (39\%) ou mixte (41\%). Le tableau 7 présente, pour chaque domaine d'intérêt couvert par la RCSMC, la proportion d'articles empiriques où une source de financement est indiquée. Mis à 


\section{Tableau 7}

Présence ou absence de mention de financement selon le domaine d'intérêt des articles empiriques

\begin{tabular}{lrr}
\hline & \multicolumn{2}{c}{ Financement } \\
\cline { 2 - 3 } & Oui & Non \\
& $n=116$ & $n=153$ \\
\hline Organisation des services sociaux $(n=12)$ & $25 \%$ & $75 \%$ \\
Évaluation de programme $(n=64)$ & $47 \%$ & $53 \%$ \\
Analyse des besoins de la communauté $(n=22)$ & $41 \%$ & $59 \%$ \\
Développement communautaire $(n=8)$ & $25 \%$ & $75 \%$ \\
Consultation $(n=0)$ & 0 & 0 \\
Action sociale $(n=33)$ & $42 \%$ & $58 \%$ \\
Entraide $(n=6)$ & $50 \%$ & $50 \%$ \\
Éducation communautaire et formation $(n=2)$ & $100 \%$ & 0 \\
Défense de droits et d'intérêts $(n=6)$ & 0 & $100 \%$ \\
Réaction à un phénomène donné $(n=37)$ & $41 \%$ & $59 \%$ \\
Autres $(n=79)$ & $48 \%$ & $52 \%$ \\
\hline
\end{tabular}

part les catégories « entraide » et « éducation communautaire et formation » qui s'appliquent à un très petit nombre d'articles, ce sont les études inclues dans la catégorie « autre », suivies de près par les évaluations de programme, qui semblent les plus financées. Elles sont suivies par les études sur l'action sociale, sur l'analyse des besoins et sur une réaction à un phénomène donné.

Entre 1982 et 1986, seulement 19\% des recherches empiriques publiées dans la RCSMC mentionnent une source de financement, alors que pour les autres périodes cette proportion est de $50 \%$ environ. La figure 4 illustre cette tendance.

\section{Figure 4}

Présence de mention de financement pour les articles empiriques par tranche de 5 ans

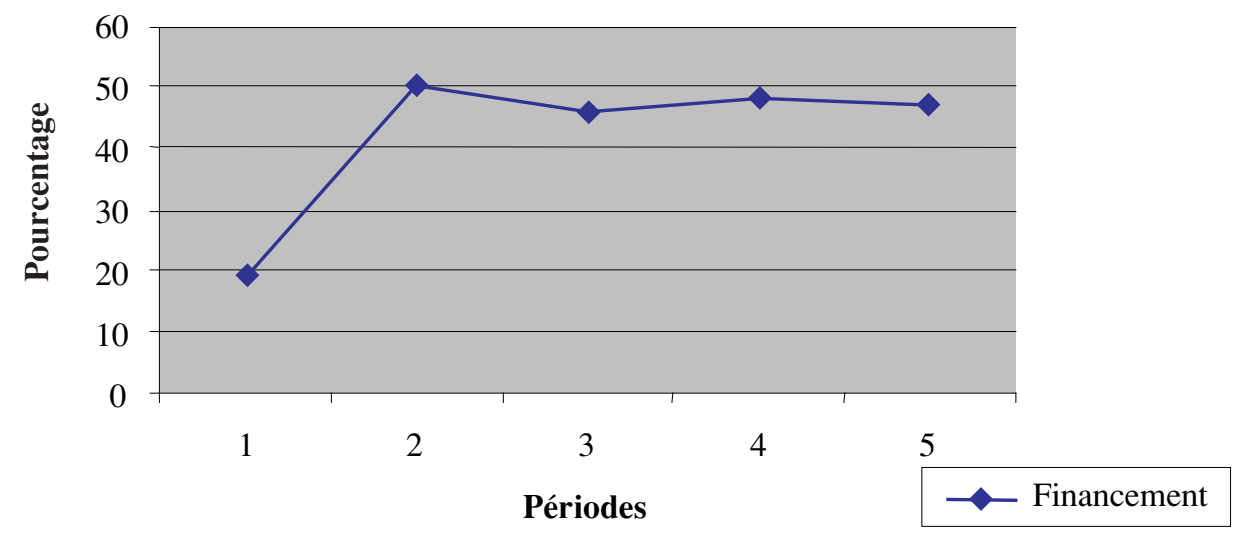

Note. $1=1982-86 ; 2=1987-91 ; 3=1992-96 ; 4$ = 1997-2001; 5 = 2002-6. 


\section{DISCUSSION}

Les résultats de l'analyse de contenu quantitative rapportés dans les pages précédentes permettent de faire divers constats sur le « qui » et le «quoi» de la RCSMC. Sur le plan du « qui », on constate que les personnes les plus influentes pour la Revue pendant les 25 dernières années (rédacteurs et rédactrices, auteurs les plus productifs) sont des universitaires, autant des femmes que des hommes. Ils proviennent de diverses universités situées surtout au Québec et en Ontario, et dans une moindre mesure dans les provinces des Prairies et en Colombie-Britannique. De plus, les proportions d'auteurs anglophones et francophones reflètent celles de la population canadienne. Sur le plan du « quoi », on constate que les articles empiriques sont prédominants et que leur proportion est en croissance depuis les 5 dernières années, alors que la proportion d'articles théoriques est nettement en baisse. Parmi les articles empiriques, ceux qui rapportent les résultats d'études quantitatives sont en baisse, au profit de ceux qui emploient une méthodologie qualitative. En ce qui concerne les domaines d'intérêt des articles, le résultat le plus frappant est que le quart de la production de la RCSMC ne se classe dans aucune des domaines spécifiés dans la mission de la Revue. Les autres domaines sont représentés de manière très inégale, et cette représentation varie dans le temps. Enfin, depuis la période débutant en 1987, des mentions de financement sont présente dans environ la moitié des articles empiriques.

Les résultats suggèrent que la RCSMC relève le défi de la diversité, du moins en partie. L'équité s'observe notamment sur le plan du genre des rédacteurs et rédactrices et des auteurs les plus productifs. Rappelons toutefois que les données disponibles n'ont pas permis de vérifier si ce constat s'applique à l'ensemble des auteurs ayant publié dans la Revue, ce qui constitue une limite importante de l'analyse en matière de genre. L'équité s'observe également sur le plan de la langue de publication. L'anglais est la langue la plus utilisée par les auteurs, mais la proportion des articles en français $(25,7 \%)$ est comparable à la proportion de francophones au Canada (22,61\%) (Statistique Canada, 2001). Bien que les auteurs francophones aient été sous-représentés durant les 5 premières années de la RCSMC, la proportion des articles francophones s'est ensuite maintenue à un pourcentage représentatif de la proportion de francophones au Canada. Il apparaît donc légitime que la RCSMC s'identifie comme une revue bilingue (Revue canadienne de santé mentale communautaire, 2001). L'équité est moins apparente en ce qui a trait à la provenance des premiers auteurs et des rédacteurs et rédactrices. On note une nette prépondérance de l'Ontario et du Québec, ce qui n'est pas surprenant vu que ces deux provinces ont un poids démographique important à l'intérieur du Canada. Toutefois, une meilleure représentation des autres provinces au sein de l'équipe éditoriale, notamment les provinces de l'Atlantique, serait à promouvoir dans les prochaines années.

Sur le plan de la discipline des personnes les plus influentes, on remarque une surreprésentation des psychologues. Ceci pourrait refléter la longue tradition empirique ce cette discipline. Il est également possible que la RCSMC réponde particulièrement à un besoin pour les psychologues d'orientation communautaire, étant donné que peu de revues scientifiques en psychologie favorisent cette perspective. Le service social apparaît comme la seconde discipline en importance en matière d'influence sur la RCSMC, alors que les autres disciplines sont très peu représentées. Rappelons toutefois que les données disponibles n'ont pas permis de vérifier si ces prédominances disciplinaires s'appliquent à l'ensemble des auteurs ayant publié dans la Revue. 
Les milieux universitaires constituent les principales affiliations institutionnelles des premiers auteurs des articles publiés dans la RCSMC. Même les articles communautaires pratiques sont majoritairement rédigés par un premier auteur universitaire. Ceci concorde avec les résultats de la première analyse de contenu de la RCSMC (Peirson \& Walsh-Bowers, 1993). Comme ces auteurs le suggèrent, ces résultats peuvent s'expliquer par une certaine pression à publier qui prévaut dans les milieux universitaires. Ils peuvent également refléter la plus grande opportunité de publier dont disposent les universitaires du fait que la publication est reconnue dans leur charge de travail, ce qui n'est pas le cas des professionnels et professionnelles. Bien que compréhensible, cette réalité est paradoxale pour la RCSMC, qui se veut «un lieu d'échange [pour les] Canadiens appartenant aux milieux tant académiques que professionnels » (Revue canadienne de santé mentale communautaire, 2001).

Les articles les plus susceptibles d'être rédigés par des praticiens et praticiennes sont ceux qui abordent des contenus pratiques. Ces articles comptent parmi les moins nombreux dans la RCSMC. ${ }^{1}$ Ceci reflète le fait que la RCSMC se définit comme une revue scientifique et que les comités de pairs qui évaluent les manuscrits sont en grande majorité composés d'universitaires. Les critères de qualité scientifique peuvent être difficiles à rencontrer pour des professionnelles et professionnels peu formés à la recherche et peu au fait des exigences de rigueur propres à la publication. Une avenue possible pour accroître le nombre de professionnels et professionnelles auteurs serait de publier en collaboration avec des chercheurs, comme co-auteurs, lorsque c'est possible. Cela permettrait d'intégrer une perspective plus pratique au contenu de la RCSMC, de promouvoir le travail en partenariat et de reconnaître l'apport des professionnels et professionnelles aux travaux de recherche et aux réflexions théoriques.

Parmi les articles de type empirique, ceux qui rapportent des recherches quantitatives sont majoritaires en nombre. Cela peut s'expliquer par le statut traditionnellement dominant des méthodes quantitatives dans certaines disciplines. En effet, les critères de fidélité et de validité propres à la recherche quantitative ont souvent servi de référents pour juger de la rigueur de la recherche qualitative, qui est alors considérée moins scientifique (Bickman \& Rog, 1998). Le fait que les études qualitatives soient moins publiées que les études quantitatives dans plusieurs domaines (Arredondo, Rosen, Rice, Perez, \& Tovar-Gamero, 2005; Fade, 2003; Kidd, 2002; Pope-Davis, Ligiero, Liang, \& Codrington, 2001) peut être attribuable à cette tendance. Par ailleurs, il importe de nuancer ce résultat en tenant compte de l'augmentation constante des publications de recherches qualitatives dans la RCSMC, parallèlement à la diminution du nombre de publications rapportant des recherches quantitatives. Ces deux tendances contraires ont fait en sorte que la publication d'études qualitatives est devenue égale en nombre à celle d'études quantitatives entre 2002 et 2006. Cela reflète sans doute la popularité et la reconnaissance croissante des méthodes qualitatives qui comportent de nombreux avantages pour la recherche en santé mentale communautaire. Le type de questions et d'outils de collectes de données utilisés en recherche qualitative permettent aux participants et participantes de se définir dans leurs propres mots (Banyard \& Miller, 1998), puisque ces outils ont été conçus afin de faire entendre les voix des groupes défavorisés socialement (Rudkin, 2003). Cela rejoint des valeurs fondamentales de la santé mentale communautaire, soit la participation et la justice (Liégeois \& Audenhove, 2005). 
Une proportion élevée d'articles publiés dans la RCSMC ne se relie à aucun des domaines d'intérêt identifiés dans sa mission. L'analyse qualitative du contenu de ces articles dépassait les objectifs de la présente étude. Une telle analyse pourrait faire l'objet d'une étude ultérieure, qui permettrait de revoir les frontières de l'expression santé mentale communautaire et d'en proposer une définition plus moderne. Si ces articles ont été publiés dans la RCSMC, c'est que des évaluateurs et évaluatrices indépendantes, de même qu'un rédacteur ou rédactrice, ont convenu que la problématique abordée était pertinente et conforme aux orientations de la Revue. Une analyse en profondeur de leur contenu permettrait notamment à la RCSMC d'augmenter la concordance entre son contenu et l'énoncé de sa mission.

Parmi les articles en lien à un domaine d'intérêt énoncé par la mission, l'évaluation de programme constitue le thème le plus souvent couvert. Ces dernières années et de façon générale, la parution d'écrits sur l'évaluation de programme a augmenté (Feldman, 2004). Selon Liégeois et Audenhove (2005), la place des évaluations de programmes en santé mentale communautaire s'explique par l'importance croissante que les décideurs accordent à l'efficacité et à l'efficience des interventions afin de s'assurer que les subventions sont dépensées en fonction de ces critères. Ce constat peut contribuer à expliquer son importance au sein de la RCSMC.

Le thème de la consultation est le moins abordé parmi les domaines d'intérêt énoncés dans la mission. La consultation constitue un type d'intervention amenant un professionnel ou une professionnelle à interagir avec une personne, un groupe, une organisation ou une communauté dans le but de modifier une situation en exerçant différents rôles et en utilisant des stratégies appropriées (Laprise \& Payette, 2001). Une étude antérieure a montré que, de façon générale, moins de cinq recherches ont été publiées dans ce domaine chaque année entre 1961 et 1989. Parmi les études parues sur la consultation pendant cette période, celles portant sur la santé mentale sont rares, particulièrement entre 1975 et 1989 (Fuchs, Fuchs, Dulan, Roberts, \& Fertstrom, 1992). Il s'agit pourtant d'une activité professionnelle largement pratiquée, dans plusieurs milieux, notamment ceux associés à la santé mentale communautaire (Laprise \& Payette, 2001). De plus, elle est reliée à des valeurs communautaires fondamentales, soit celles de l'autonomie qui est visée pour l'organisation recevant la consultation et du soutien apporté par cette pratique (Liégeois \& Audenhove, 2005). De fait, le modèle de consultation axé sur les processus, souvent utilisé en milieu communautaire, constitue un soutien du milieu dans lequel la consultation se fait en vue d'une plus grande autonomie.

Le peu de publications sur ce domaine d'intérêt peut s'expliquer par le fait qu'il s'agit essentiellement d'une activité professionnelle et que les professionnels et professionnelles publient peu dans la RCSMC. La consultation est exercée par des professionnels et professionnelles souvent pour d'autres professionnels et professionnelles (Laprise \& Payette, 2001; Lescarbeau, Payette, \& StArnaud, 2003). La recherche à ce sujet pose plusieurs difficultés pour les chercheurs. Ces recherches impliquent une importante planification avec un grand nombre d'actrices et d'acteurs du milieu. Elles supposent l'observation de la pratique des consultants et consultantes, de leur effet sur les professionnels et professionnelles et de la pratique subséquente de ces professionnels et professionnelles auprès de la population. La recherche à ce sujet, en supposant l'observation d'effets indirects, s'avère ainsi plus complexe que la recherche sur d'autres types d'intervention (Fuchs et al., 1992). 
Malgré qu'un numéro spécial de la RCSMC ait porté sur les groupes d'entraide et les groupes de soutien, l'entraide est le deuxième domaine d'intérêt le moins couvert par les articles. Cela peut s'expliquer par le fait que les groupes d'entraide, principal sujet couvert par les articles sur l'entraide, excluent par définition les professionnels et professionnelles. De fait, les groupes d'entraide sont autogérés par les personnes concernées par une difficulté donnée (Lavoie, 2001). Dans ce contexte, les professionnels et professionnelles et les chercheurs ne sont pas souvent en mesure d'observer ces groupes de l'intérieur. Par ailleurs, plusieurs difficultés méthodologiques ont été identifiées relativement à l'étude de l'impact de ces groupes sur leurs membres. Notamment, le suivi des participants et participantes se fait difficilement puisque certains et certaines peuvent partir ou arriver en cours de route. De plus, ces groupes se recréent difficilement en contexte expérimental (Lavoie \& Dufort, 1995).

Après l'entraide et la consultation, le domaine d'intérêt le moins couvert par les articles publiés est la défense de droits et d'intérêts. Le mouvement social de défense de droits et d'intérêts des personnes psychiatrisées est pourtant identifié comme un acteur clé dans l'émergence de l'approche de la santé mentale communautaire (Rosenberg \& Rosenberg, 2006). En 1963, aux États-Unis, John F. Kennedy mettait de l'avant le Community Mental Health Act et déclarait que les inégalités sociales étaient à la base des problèmes de santé mentale. Cette loi a soutenu financièrement l'implantation des Community Mental Health Centers (Feldman, 2004; Kiely \& Moreau, 1975). Encore aujourd'hui, la défense de droits et d'intérêts constitue une préoccupation importante en santé mentale communautaire. La justice, qui renvoie à la distribution équitable des ressources dans la société, et la solidarité, qui réfère à la préoccupation pour autrui et au bien commun, font partie des valeurs fondamentales de la santé mentale communautaire selon Liégeois et Audenhove (2005).

Dans la majorité des recherches empiriques publiées dans la RCSMC, il n'est pas fait mention de source de financement, même si elles sont conduites par des personnes affiliées à une université. Ce fait est étonnant, considérant que la recherche implique des coûts parfois importants. Il est toutefois difficile de savoir si les recherches rapportées ne sont effectivement pas subventionnées, ou si cet élément n'est pas spécifié systématiquement par les auteurs. En effet, il se peut qu'il y ait des données absentes relativement à cette dimension de l'analyse.

\section{FORCES ET LIMITES DE L'ANALYSE}

La présente analyse de contenu comporte certaines limites qu'il importe de mentionner. Étant donné que seuls les résumés et certaines informations factuelles ont fait l'objet de l'analyse de contenu quantitative, certaines subtilités plus qualitatives ont forcément échappé à l'analyse. Ce choix, dicté par des impératifs de faisabilité, a limité les éléments investigués et la profondeur de l'analyse. Malgré cela, les dimensions couvertes par l'analyse sont nombreuses et diversifiées et permettent de dresser un portrait global des publications. Il a également été possible d'identifier des tendances dans le temps pour les éléments étudiés. Bref, l'exhaustivité a été privilégiée à l'analyse détaillée.

D'autres limites sont attribuables au fait que certaines dimensions n'ont pas été analysées. Par exemple, le fait d'avoir pris en compte les affiliations institutionnelles des premiers auteurs seulement 
limite certaines interprétations de résultats. Il est possible que la proportion des auteurs ayant une affiliation institutionnelle non universitaire ait été différente si tous les auteurs avaient été pris en considération dans l'analyse. Ensuite, le fait de ne pas avoir disposé de l'information relativement au genre et aux disciplines des différents auteurs a empêché d'évaluer pleinement l'équité hommes-femmes et le caractère interdisciplinaire de la RCSMC. Une autre limite de l'étude est que les trois analystes étudient à la même université, l'Université Laval, et dans le même domaine, la psychologie communautaire. Ainsi, l'homogénéité des analystes ne reflète pas le caractère national et interdisciplinaire de la RCSMC. Par contre, cette limite a eu peu d'influence sur la collecte des données puisque la majorité des données recueillies étaient factuelles, donc non sujettes à des biais. Le fait que les analystes soient rattachées à la même université a facilité une collaboration constante entre elles tout au long de l'analyse. Enfin, le fait qu'elles soient bilingues a facilité l'analyse équitable de tous les textes publiés depuis 1982 .

\section{CONCLUSION}

Suite à cette analyse de contenu, plusieurs pistes de réflexion peuvent être formulées pour la RCSMC. Un effort pourrait être consacré pour accroître l'influence de certaines disciplines ou régions sur la Revue et la visibilité des domaines d'intérêt les moins couverts, soit la consultation, l'entraide et la défense de droits et d'intérêts, dans la mesure où la RCSMC désire conserver ces domaines d'intérêt dans sa mission. Cela pourrait être fait par l'intermédiaire de numéros spéciaux futurs consacrés à ces domaines d'intérêt. De plus, un meilleur soutien aux publications provenant de milieux de pratique pourrait permettre d'augmenter le nombre d'articles sur certains domaines d'intérêt moins couverts. Il serait également opportun de réfléchir à la concordance entre les domaines d'intérêt énoncés dans la mission et le contenu des publications de la RCSMC. Pour pallier à certaines limites de la présente analyse, il serait pertinent de conserver à l'avenir des informations relatives à la discipline et au genre des auteurs. Dans le cadre d'analyses futures, il serait intéressant de prendre ces dimensions en compte et de traiter plus systématiquement la provenance géographique des auteurs, afin de pouvoir généraliser davantage les résultats sur ces aspects.

Au cours des dernières années, un vent de modernisme a soufflé sur la RCSMC, qui est notamment devenue une revue électronique depuis le printemps 2006. D'autres changements sont certainement à envisager dans les 25 prochaines années. Il reste à souhaiter que la présente analyse serve de pierre d'assise à la réflexion des prochaines équipes de rédaction et constitue un outil de « monitoring » utile à cette publication unique au Canada.

\section{NOTE}

1. Il convient toutefois de souligner que certains articles empiriques comprennent une description de pratiques, comme les évaluations de programmes par exemple. 


\section{REVUE CANADIENNE DE SANTÉ MENTALE COMMUNAUTAIRE}

\section{RÉFÉRENCES}

Arredondo, P., Rosen, D.C., Rice, T., Perez, P., \& Tovar-Gamero, Z.G. (2005). Multicultural counseling: A 10years content analysis of the Journal of Counseling \& Development. Journal of Counseling \& Development, 83, 155-161.

Austin, C.D., Des Camp, E., Flux, D., McClelland, R.W. \& Sieppert, J. (2005). Community development with older adults in their neighborhoods: The elder friendly communities program. Families in Society: The Journal of Contemporary Social Services, 86, 401-409.

Banyard, V.L., \& Miller, K.E. (1998). The powerful potential of qualitative research for community psychology. American Journal of Community Psychology, 26, 485-506.

Bennett, E.M., \& Trute, B. (1982). Editorial statement. Revue canadienne de santé mentale communautaire, 1, 2-4.

Bickman, L., \& Rog, D.J. (1998). Handbook of applied social research methods. Thousand Oaks, CA: Sage.

Buboltz, W.C., Miller, M., \& Williams, D.J. (1999). Content analysis of research in the Journal of Counseling Psychology (1973-1998). Journal of Counseling Psychology, 46, 496-503.

Buboltz, W., \& Savickas, M. (1994). A 20-year retrospective of the Career Development Quarterly. Career Development Quarterly, 42, 367-381.

Cokley, K., Caldwell, L., Miller, K., \& Muhammad, G. (2001). Content analysis of the Journal of Black Psychology (1985-1999). Journal of Black Psychology, 27, 424-438.

Dufort, F. (2004). Évaluation psychosociale des milieux. Présentation dans le cours «Évaluation psychosociale des milieux ». Sainte-Foy, QC: Université Laval.

Fade, S.A. (2003). Communicating and judging the quality of qualitative research: The need for a new language. Journal of Nutrition and Dietetics, 16, 139-149.

Feldman, S. (2004). Reflections on the 40th anniversary of the Community Mental Health Centers Act. Administration and Policy in Mental Health, 31, 369-380.

Fuchs, D., Fuchs, L., Dulan, J., Roberts, H., \& Fernstrom, P. (1992). Where is the research on consultation effectiveness? Journal of Educational and Psychological Consultation, 3, 151-174.

Howard, G.S., Cole, D.A., \& Maxwell, S.E. (1987). Research productivity in psychology based on publications in the journals of the American Psychological Association. American Psychologist, 42, 975-986.

Kidd, S.A. (2002). The role of qualitative research in psychological journals. Psychological Methods, 7, $126-138$.

Kiely, M.C., \& Moreau, D. (1975). Psychologie communautaire. Courrier-cours, 2, 1-14.

Laprise, R., \& Payette, M. (2001). Le choix d'un modèle de consultation selon une perspective communautaire. Dans F. Dufort \& J. Guay (dir.), Agir au cour des communautés: la psychologie communautaire et le changement social (pp. 187-216). Saint-Nicolas, QC: Presses de l'Université Laval.

Lavoie, F. (2001). Les groupes de soutien et les groupes d'entraide. Dans F. Dufort \& J. Guay (dir.), Agir au cœur des communautés: la psychologie communautaire et le changement social (pp. 157-181). Saint-Nicolas, QC: Presses de l'Université Laval.

Lavoie, F., \& Dufort, F. (1995). La recherche avec les groupes d'entraide: les difficultés méthodologiques et les défis. Revue canadienne du vieillissement, 14, 177-200.

L'Écuyer, R. (1990). Méthodologie de l'analyse développementale de contenu: méthode GPS et concept de soi. Sillery, QC: Presses de l'Université du Québec.

Lescarbeau, R., Payette, M., \& St-Arnaud, Y. (2003). Profession: consultant. Montréal: Gaëtan Morin.

Liégeois, A., \& Audenhove, C.V. (2005). Ethical dilemmas in community mental health care. Journal of Medical Ethics, 31, 452-456.

Loveland, J.M., Buboltz, W.C., Schwartz, J., \& Gibson, G. (2006). Content analysis of CDQ from 1994-2003: Implications and trends for practitioners and researchers from a decade of research. Career Development Quarterly, 54, 256-264.

Morency, J., Couture, C., Johnson, C., \& Kiely, M.C. (1988). Vers la conceptualisation d'un modèle d'intervention en psychologie. Revue canadienne de santé mentale communautaire, 7, 81-88.

Mucchielli, A. (1996). Dictionnaire des méthodes qualitatives en sciences humaines et sociales. Paris: Armand Colin. 


\section{ANALYSE DE CONTENU}

Novaco, R.W., \& Monahan, J. (1980). Research in community psychology: An analysis of work published in the first six years of the American Journal of Community Psychology. American Journal of Community Psychology, 8, 131-145.

Office québécois de la langue française (2002). Grand dictionnaire terminologique. Québec: Gouvernement du Québec. Récupéré en ligne le 5 janvier 2007 de: http://www.granddictionnaire.com/btml/fra/r_motclef/ index1024_1.asp

Ouattara, C. (2003, juin). Développement communautaire et réduction de la pauvreté dans un contexte de décentralisation. Communication Séminaire ENDSA. Récupéré en ligne le 5 janvier 2007 de: http:// unpan1.un.org/intradoc/groups/public/documents/UN/UNPAN011027.pdf

Peirson, L., \& Walsh-Bowers, R. (1993). The first decade of the Canadian Journal of Community Mental Health: A critical review. Revue canadienne de santé mentale communautaire, 12(1), 23-35.

Pope-Davis, D.B., Ligiero, D.P., Liang, C., \& Codrington, J. (2001). Fifteen years of the Journal of Multicultural Counseling and Development: A content analysis. Journal of Multicultural Counseling and Development, 29, 226-238.

Posavac, E.J., \& Carey, R.G. (2003a). The assessment of need. Dans E.J. Posavac \& R.G. Carey (dir.), Program evaluation: Methods and case studies (pp. 114-131). Upper Saddle River, NJ: Prentice Hall.

Posavac, E.J., \& Carey, R.G. (2003b). Program evaluation: An overview. Dans E.J. Posavac \& R.G. Carey (dir.), Program evaluation: Methods and case studies (pp. 1-22). Upper Saddle River, NJ: Prentice Hall.

Revue canadienne de santé mentale communautaire. (2001). Manual for the Canadian Journal of Community Mental Health. Waterloo, ON: Auteur.

Rosenberg, J., \& Rosenberg, S. (2006). Introduction conceptualizing the challenges in community mental health. Dans J. Rosenberg \& S. Rosenberg (dir.), Community mental health: Challenges for the 21st century (pp. 1-6). New York: Routledge.

Rudkin, J.K. (2003). Community psychology: Guiding principles and orienting concepts. Upper Saddle River, NJ: Prentice Hall.

Statistique Canada. (2001). Langue maternelle détaillée (160), sexe (3), connaissance des langues officielles (5) et groupes d'âge (15) pour la population, pour le Canada, les provinces, les territoires, les régions métropolitaines de recensement et les agglomérations de recensement, recensements de 1996 et $2001-$ Données-échantillon (20\%). Ottawa: Gouvernement du Canada.

Wakefield, S.E.L., \& Poland, B. (2005). Family, friend or foe?: Critical reflections on the relevance and role of social capital in health promotion and community development. Social Science \& Medicine, 60(12), 28192832. 


\section{APPENDICES}

\section{Grille de codification}

\begin{tabular}{|c|c|}
\hline Année & \\
\hline Volume & \\
\hline Numéro & \\
\hline Titre de l'article & \\
\hline Pages & \\
\hline Auteur-e-s & \\
\hline $\begin{array}{l}\text { Affiliation institutionnelle } \\
1^{\text {er-ère }} \text { auteur-e }\end{array}$ & \\
\hline Langue de l'article & Français \\
\hline Type d'article & Empirique, théorique, recension d'écrits, communautaire pratique \\
\hline $\begin{array}{l}\text { Si empirique, type de } \\
\text { méthodologie: }\end{array}$ & $\begin{array}{l}\text { - qualitatif } \\
\text { - quantitatif } \\
\text { - mixte (qualitatif et quantitatif) }\end{array}$ \\
\hline $\begin{array}{l}\text { Domaine d'intérêt énoncé dans la } \\
\text { mission de la RCSMC: }\end{array}$ & $\begin{array}{l}\text { L'organisation des services sociaux, l'évaluation de programmes, } \\
\text { l'analyse des besoins de la communauté, le développement } \\
\text { communautaire, l'action sociale, l'entraide, l'éducation communautaire et } \\
\text { la formation, la consultation et la défense de droits et d'intérêts. Préciser } \\
\text { si autre. }\end{array}$ \\
\hline $\begin{array}{l}\text { Mention de financement des } \\
\text { recherches empiriques publiées: }\end{array}$ & Oui Non \\
\hline
\end{tabular}


ANALYSE DE CONTENU

\section{Définitions des types d'articles}

Communautaire pratique: L'article décrit une expérience de mise en œuvre de programme ou d'intervention dans le domaine de la santé mentale communautaire (Novaco \& Monahan, 1980; Peirson \& Walsh-Bowers, 1993).

Empirique: L'article rapporte des données afin de répondre à des questions posées par les auteurs (Novaco \& Monahan, 1980). Un article empirique peut être quantitatif, lorsque les données sont numériques; qualitatif, lorsque les données ne sont pas numériques; ou mixte, lorsque les deux types de données sont présents.

Recension d'écrits: L'article présente une revue systématique des idées et des résultats de recherches sur un sujet spécifique. De nouvelles idées peuvent être introduites, mais l'accent premier est sur la recension des écrits (Novaco \& Monahan, 1980).

Théorique: L'article introduit de nouvelles idées ou concepts ou élabore sur des idées et concepts déjà existants (Peirson \& Walsh-Bowers, 1993). Il met l'accent sur certains enjeux relativement à ces idées ou concepts. Il peut référer à d'autres théories et recherches pour mettre en contexte ces idées et concepts. Par contre, l'accent premier n'est pas mis sur la recension d'écrits, mais sur les idées et concepts nouveaux ou élaborés (Novaco \& Monahan, 1980).

\section{Définitions des thèmes relatifs à la mission}

Action sociale: Forme de développement communautaire (Wakefield \& Poland, 2005) mettant de l'avant un ensemble de mesures collectives visant à mettre en œuvre une politique de solidarité dans le domaine social (Office québécois de la langue française, 2002).

Analyse des besoins de la communauté: Examen détaillé des éléments essentiels (Dufort, 2004) que les gens doivent avoir pour se trouver dans un état satisfaisant (Posavac \& Carey, 2003a).

Autres: Impossible à classer dans une des catégories existantes.

Consultation: Type d'intervention amenant un professionnel ou une professionnelle à interagir avec une personne, un groupe, une organisation ou une communauté dans le but de modifier une situation en exerçant différents rôles et en utilisant des stratégies appropriées (Laprise \& Payette, 2001).

Défense de droits et d'intérêts: Promotion de l'amélioration des conditions de vie des groupes défavorisés socialement et du respect de leurs droits fondamentaux en vue de l'atteinte d'une plus grande justice sociale.

Développement communautaire: Démarche volontaire d'actrices et d'acteurs souvent marginalisés (Austin, Des Camp, Flux, McClelland, \& Sieppert, 2005) œuvrant à l'amélioration de leurs conditions 
de vie dans leurs localités notamment par le développement d'activités relatives à la santé, à l'éducation et à l'approfondissement de la démocratie locale (Ouattara, 2003).

Éducation communautaire et formation: Ensemble des actions susceptibles d'enrichir les connaissances d'une communauté, de développer ses aptitudes déterminant le comportement dans les différentes situations dans lesquelles elle se trouve au cours de sa vie (Office québécois de la langue française, 2002).

Entraide: Action réciproque d'aide et de soutien.

Évaluation de programme: Ensemble de méthodes et d'habiletés utiles pour déterminer si un service humain est nécessaire et susceptible d'être utilisé, si le service est offert de façon suffisante pour répondre aux besoins identifiés, s'il est offert tel que prévu et si le service aide à rencontrer les besoins à un coût raisonnable sans effet secondaire inacceptable (Posavac \& Carey, 2003b).

Organisation des services sociaux: Groupement, régi ou non par des institutions, qui se propose des buts déterminés (Office québécois de la langue française, 2002) dans le domaine de la santé et des services sociaux.

Réaction à un phénomène donné: Attitude d'une personne ou d'un groupe face à un événement ou à l'action de quelqu'un d'autre ou d'un groupe (Office québécois de la langue française, 2002). 